\title{
Conditional probabilities for a single photon at a beam splitter
}

\author{
K. Jacobs and P. L. Knight \\ Optics Section, The Blackett Laboratory, Imperial College, London SW7 2BZ, England
}

(Received 16 May 1996; revised manuscript received 25 July 1996)

\begin{abstract}
Eight-port homodyne detection may be used to perform a joint measurement on a single-mode input to a beam splitter; the $x$ quadrature is measured at one output port, and the $p$ quadrature is measured at the other. This is possible even when the input mode contains only one photon. We show to what extent this "joint measurement of a single photon" may be reconciled with the semiclassical notion that the photon may only exit from one of the beam splitter output ports. [S1050-2947(96)50311-2]
\end{abstract}

PACS number(s): 42.50.Dv, 03.65.Bz

In a standard eight-port homodyne measurement scheme the light field to be measured is split into two with a beam splitter, and each of the resulting beams is measured using homodyne detection [1-3]. The phase difference between the field quadratures measured by the two homodyne detectors is chosen to be $\pi / 2$, so that one detector may measure, for example, the $x$ quadrature, while the other measures the $p$ quadrature. The joint probability distribution for $x$ and $p$ is a scaled version of the Husimi $Q$ function for the input field [4]; if we consider just one photon incident on the initial beam splitter, then the joint probability distribution is a scaled version of the Husimi $Q$ function for one photon. That is, at both homodyne detectors we appear to gain information on the fact that there is a photon incident at the beam splitter at each measurement, even though for any given measurement we expect, from a semiclassical point of view, that the photon can only be incident at one of the homodyne detectors [5]. At first sight, the idea of a joint measurement of the conjugate variables $x$ and $p$ for a single photon using an eight-port homodyne measurement (so that $x$ is measured on the output of one port of the beam splitter and $p$ on the other port) may seem alarming. After all, it would seem that the photon can either be reflected or transmitted by the beam splitter, apparently ruling out any possibility of a joint measurement. As we will show, such alarm is at least partially unwarranted. The eight-port device is a realization of the Arthurs-Kelly joint measurement scheme [6]; what are detected are the quadratures of the beam splitter outputs, which are related to the two input ports: signal and empty port. In what follows we analyze the situation of a single photon at a beam splitter, and show that the results at each homodyne detector separately are consistent with the semiclassical view that each detector should only gain information half the time that a photon is incident at the beam splitter. We also point out, however, that when correlations between the results at each homodyne detector are considered, the quantum nonlocality of a single photon appears, and it is not possible to view the photon as having "really" taken just one of the two possible paths. We note that Grice and Walmsley [7] have recently considered a single photon incident on a beam splitter, with homodyne detection performed on each output, although they focus on a different aspect of the situation from that which we discuss here.

Before we begin, let us review briefly the results for the joint measurement of the single-mode field quadratures $\hat{x}=\left(a+a^{\dagger}\right) / 2$ and $\hat{p}=-i\left(a-a^{\dagger}\right) / 2$ in an eight-port homodyne detection scheme. The $Q$ function, which describes the state of the input field, is defined by

$$
Q(x, p)=\frac{1}{\pi}\langle x+i p|\rho| x+i p\rangle,
$$

where $\rho$ is the density matrix for the input field and $|x+i p\rangle$ is a coherent state with complex amplitude $\alpha=x+i p$. When eight-port homodyne detection is used to measure the state of a single-mode field, the measured joint distribution, $P(x, p)$, obtained for $x$ and $p$ is given by

$$
P(x, p)=2 Q(\sqrt{2} x, \sqrt{2} p),
$$

which is a scaled version of the $Q$ function. The total distribution for $x$ alone is therefore the marginal of this scaled $Q$ function. We note here that this result has been generalized by Leonhardt and Paul [8] to the case when the initial beam splitter is not 50-50. In that case the measured joint distribution has an ordering that depends on the beam splitter reflectivity. In what follows we will denote the $Q$ function for an $n$-photon-number state as $Q_{n}$, and similarly the Wigner function for an $n$-photon-number state by $W_{n}$.

Let us examine the experimental setup depicted in Fig 1. Homodyne detection is performed on the beam at $\mathrm{A}$, and simple photon counting is performed at $\mathrm{B}$. The input beam,

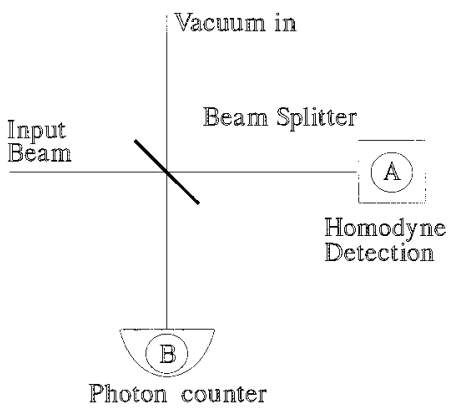

FIG. 1. A diagram of the experimental arrangement discussed in the text. The input beam is split by a beam splitter. Homodyne detection is performed on one of the resulting output beams, while photon-counting is performed on the other. In the case of eight-port homodyne detection the photon counter at B is replaced by a homodyne detector phase stabilized relative to the detector at $\mathrm{A}$. 
which is in a one-photon state, is incident at one port of the beam splitter, and the vacuum is incident at the other port. The combined state of the two beams output from the beam splitter (one of which is detected at A, and the other at B) is $(|01\rangle+|10\rangle) / \sqrt{2}[9]$. For the sake of definiteness let the first digit refer to the number of photons in beam $\mathrm{A}$, and the second to the number of photons in beam $\mathrm{B}$. We note in passing that a joint measurement on this state may be used to violate Bell's inequalities [10].

Without loss of generality, let us measure the $x$ quadrature at $\mathrm{A}$. We are therefore interested in the joint probability for a particular result for $x$ at $\mathrm{A}$, and the detection of $n$ photons at B. This is readily calculated using

$$
P(x, n)=\frac{1}{2} \mid\left\langle x, n|(|01\rangle+|10\rangle)|^{2},\right.
$$

where

$$
|x, n\rangle=\sum_{m=0}^{\infty}\langle x \mid m\rangle|m, n\rangle,
$$

and $|x\rangle$ is an eigenstate of the quadrature operator, so that

$$
\langle x \mid m\rangle=\left(\sqrt{\frac{2}{\pi}} \frac{1}{2^{m} m !}\right)^{1 / 2} H_{m}(\sqrt{2} x) e^{-x^{2}},
$$

where $H_{m}$ is the $m$ th Hermite polynomial. The result is

$$
\begin{aligned}
& P(x, 0)=\frac{1}{2}|\langle x \mid 1\rangle|^{2}, \\
& P(x, 1)=\frac{1}{2}|\langle x \mid 0\rangle|^{2} .
\end{aligned}
$$

There is no quantum interference between the paths that the photon may take, since we are able to measure at B which path was taken. The distribution for $x$, given that $n$ photons have been detected at $\mathrm{B}$, which we denote by $P(x \mid n)$, is therefore

$$
\begin{aligned}
& P(x \mid 0)=|\langle x \mid 1\rangle|^{2}, \\
& P(x \mid 1)=|\langle x \mid 0\rangle|^{2} .
\end{aligned}
$$

This result is clearly expected: if we detect no photons at B, then we have a photon at $\mathrm{A}$, and the distribution obtained for the $x$ measurements, conditioned upon the detection of no photons at $\mathrm{B}$, will be the distribution for a one-photon state. Similarly, when we obtain a photon at B, the distribution of $x$ at $\mathrm{A}$ is that for zero photons.

Using the standard result that the marginal distribution of the Wigner function for a particular variable is the probability distribution for the measurement of that variable [11], we see that the conditional distribution for $x$, when we detect a photon at $\mathrm{B}$, is the marginal of the Wigner function for zero photons, and conversely for the distribution conditioned on zero photons at B.

The total distribution obtained for $x$ at $\mathrm{A}$ is given by

$$
P(x)=P(x, 0)+P(x, 1)=\frac{1}{2} P(x \mid 0)+\frac{1}{2} P(x \mid 1),
$$

Wigner Function for $n=0$

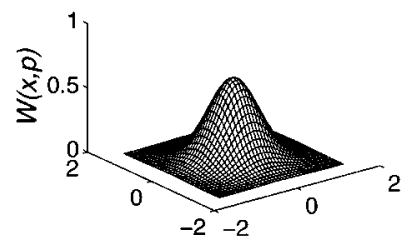

Scaled $Q$ Function for $n=0$

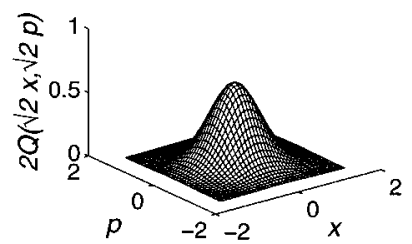

Wigner Function for $n=1$

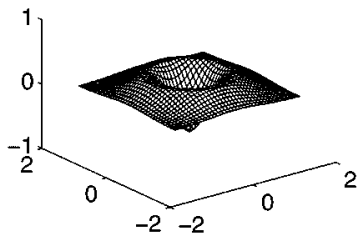

Scaled $Q$ Function for $n=1$

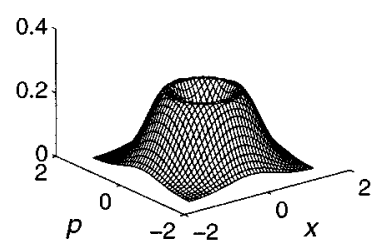

FIG. 2. The Wigner and scaled $Q$ functions for both one-photon and vacuum states. The scaled $Q$ function for the vacuum state is identical to the vacuum Wigner function. The measured joint distribution, the scaled $Q$ function for one photon, is the average of the Wigner functions for one and zero photons.

which, from the discussion above, is the average of the $x$ marginals of the Wigner functions for one and zero photons. Now, as we know that the total distribution obtained for $x$ is the marginal of the scaled $Q$ function for one photon, it follows that

$$
\begin{aligned}
P(x) & =\frac{1}{2} P(x \mid 0)+\frac{1}{2} P(x \mid 1) \\
& =2 \int Q_{1}(\sqrt{2} x, \sqrt{2} p) d p .
\end{aligned}
$$

That is, the marginal of the scaled one-photon $Q$ function is the average of the marginals for the Wigner functions for one and zero photons. As this is true for all quadratures, not merely $x$, we may conclude that

$$
2 Q_{1}(\sqrt{2} x, \sqrt{2} p)=\frac{1}{2} W_{0}(x, p)+\frac{1}{2} W_{1}(x, p),
$$

a result that is easily checked given

$$
\begin{gathered}
W_{0}=\frac{2}{\pi} e^{-2|\alpha|^{2}}, \quad W_{1}=\frac{2}{\pi}\left(4|\alpha|^{2}-1\right) e^{-2|\alpha|^{2}}, \\
Q_{1}=\frac{1}{\pi}|\alpha|^{2} e^{-|\alpha|^{2}},
\end{gathered}
$$

and $x=\operatorname{Re}[\alpha], \quad p=\operatorname{Im}[\alpha]$. The fact that it is the $Q$-function marginal that is obtained with this measurement setup, rather than the Wigner function, keeps the results consistent with the view that each detector only obtains information regarding the incidence of a photon at the beam splitter on average half the time. We plot both the Wigner and $Q$ functions for one and zero photons in Fig 2. Note that the scaled $Q$ function for the vacuum is identical to the vacuum Wigner function, which also follows from our argument above. 
This argument may be readily extended to the situation when more than one photon is incident at the initial beam splitter. If the input beam contains $n$ photons, then these will distribute themselves between the two output ports. In any one measurement, anywhere between zero and $n$ photons will arrive at A, and as we know that the total distribution for a quadrature detected at A is the marginal of the scaled $Q$ function for $n$ photons, the scaled $Q$ function for $n$ photons must be a weighted sum of the Wigner functions for zero to $n$ photons. The weight for $W_{m}$ will be given by the probability for $n-m$ photons to be detected at B.

We turn now to the situation in which homodyne detection is performed at both $\mathrm{A}$ and $\mathrm{B}$. We will denote the annihilation operator for the mode measured at A by $a$, and for that measured at B by $b$. Let us define the rotated quadrature operators $\hat{x}_{\theta}$ and $\hat{y}_{\phi}$ by

$$
\begin{gathered}
\hat{x}_{\theta}=\frac{1}{2}\left(a e^{i \theta}+a^{\dagger} e^{-i \theta}\right)=\hat{x} \cos \theta-\hat{p} \sin \theta, \\
\hat{y}_{\phi}=\frac{1}{2}\left(b e^{i \phi}+b^{\dagger} e^{-i \phi}\right),
\end{gathered}
$$

the eigenstates of which are

$$
\begin{array}{cc}
\hat{x}_{\theta}|x, \theta\rangle=x|x, \theta\rangle, & |x, \theta\rangle=e^{-i a^{\dagger} a \theta}|x\rangle, \\
\hat{y}_{\phi}|y, \phi\rangle=y|y, \phi\rangle, & |y, \phi\rangle=e^{-i b^{\dagger} b \phi}|y\rangle .
\end{array}
$$

We will set the detector at A to measure $\hat{x}_{\theta}$, and that at B to measure $\hat{y}_{\phi}$. First let us examine the results we would obtain for the joint measurement of $\hat{x}_{\theta}$ and $\hat{y}_{\phi}$ if we made the semiclassical assumption that the single photon was either reflected or transmitted by the beam splitter. In this case there is a one-half probability that we will obtain the one-photon distribution for $\hat{x}_{\theta}$ at $\mathrm{A}$ and the zero-photon distribution for $\hat{y}_{\phi}$ at $\mathrm{B}$, and a one-half probability for the reverse case. The joint probability distribution for the measurement of $\hat{x}_{\theta}$ and $\hat{y}_{\phi}$ under these assumptions is therefore

$$
\begin{aligned}
P(x, \theta ; y, \phi)= & \frac{1}{2}|\langle 1 \mid x, \theta\rangle|^{2}|\langle 0 \mid y, \phi\rangle|^{2} \\
& +\frac{1}{2}|\langle 0 \mid x, \theta\rangle|^{2}|\langle 1 \mid y, \phi\rangle|^{2} .
\end{aligned}
$$

Using the expression

$$
\begin{aligned}
\langle m \mid x, \theta\rangle & =\left\langle m\left|e^{-i \theta a^{\dagger} a}\right| x\right\rangle \\
& =\left(\sqrt{\frac{2}{\pi}} \frac{1}{2^{m} m !}\right)^{1 / 2} H_{m}(\sqrt{2} x) e^{i m \theta-x^{2}},
\end{aligned}
$$

this is easily evaluated to obtain

$$
P(x, \theta ; y, \phi)=\frac{4}{\pi}\left(x^{2}+y^{2}\right) e^{-2\left(x^{2}+y^{2}\right)} .
$$

However, performing the quantum calculation for the joint probability of measuring $\hat{x}_{\theta}$ and $\hat{y}_{\phi}$, we obtain

$$
\begin{aligned}
P(x, \theta ; y, \phi) & =\frac{1}{\sqrt{2}} \mid\left(\left.\langle 1,0|+\langle 0,1|) \mid x, \theta, y, \phi\rangle\right|^{2}\right. \\
& =\frac{4}{\pi}\left[x^{2}+y^{2}+2 x y \cos (\theta-\phi)\right] e^{-2\left(x^{2}+y^{2}\right)} .
\end{aligned}
$$

We see that, except for the special cases in which $\theta-\phi$ is an odd multiple of $\pi / 2$ (which is true when $x$ is measured at one output port and $p$ is measured at the other in order to obtain the $Q$ function), the quantum expression differs from the semiclassical expression, which was derived assuming that the photon "really" goes to one detector or the other. We see therefore that we cannot really view the photon as having taken only one of the two paths. The correlations between the measured values of $\hat{x}_{\theta}$ and $\hat{y}_{\phi}$, which appear in the quantum expression, are nonlocal since the measurements are performed at spatially separated points. It is these nonlocal correlations that are at the heart of the violation of Bell's inequalities [10].

In conclusion, we may say that, while the results at each detector, if viewed separately, are consistent with the view that information regarding the arrival of a photon at the beam splitter is gained at each detector only half the time, the correlations between the measurements at each detector show that, even though this is the case, we cannot view the photon as having really taken only one of the two possible paths.

One-photon wave packets have been employed in quantum-optical measurements for some time $[5,12]$ and nondegenerate optical parametric amplifiers have been used particularly in the test of fundamental principles of quantum physics [13]. A potential way to realize the measurements described in the present paper would be to use the detection of one photon from a nondegenerate down-converter to gate a beam splitter setup as shown in Fig. 1 .

We would like to thank Anton Zeilinger for discussions on joint measurements on single-photon states and Sze Tan for very helpful suggestions regarding the nonlocal correlations. This work was supported in part by the U.K. Engineering and Physical Sciences Research Council and the European Union. K.J. would like to acknowledge support from the British Council and the New Zealand Vice Chancellor's Committee.
[1] N. G. Walker and J. E. Carroll, Opt. Quantum Electron. 18, 355 (1986); N. G. Walker, J. Mod. Opt. 34, 15 (1987).

[2] The theory of eight-port homodyne detection and its relation to joint measurements is reviewed in R. Loudon and P. L. Knight, J. Mod. Opt. 34, 709 (1987).
[3] S. Stenholm, Ann. Phys. (N.Y.) 218, 233 (1992); M. Freyberger, K. Vogel, and W. P. Schleich, Phys. Lett. A 176, 41 (1993); M. Freyberger and W. Schleich, Phys. Rev. A 47, R30 (1993); M. Freyberger, K. Vogel, and W. Schleich, Quantum Opt. 5, 65 (1993); U. Leonhardt and H. Paul, Prog. Quantum 
Electron. 19, 89 (1995); M. Freyberger, M. Heni, and W. P. Schleich, Quantum Semiclass. Opt. 7, 187 (1995).

[4] H. Weyl, The Theory of Groups and Quantum Mechanics (Dover, New York, 1950); V. I. Tatarskij, Usp. Fiz. Nauk 139, 587 (1983) [Sov. Phys. Usp. 26, 311 (1983)]; M. Hillery, R. F. O'Connell, M. O. Scully, and E. P. Wigner, Phys. Rep. 106, 121 (1984); S. Abe and N. Suzuki, Phys. Rev. A 45, 520 (1992).

[5] P. Grangier, G. Roger, and A. Aspect, Europhys. Lett. 1, 173 (1986); A. Aspect and P. Grangier, Hyperfine Interact. 37, 3 (1987).

[6] E. Arthurs and J. L. Kelly, Jr., Bell Syst. Tech. J. 44, 725 (1965).

[7] W. Grice and I. A. Walmsley, J. Mod. Opt. 43, 795 (1996).

[8] U. Leonhardt and H. Paul, Phys. Rev. A 48, 4598 (1993).

[9] D. F. Walls and G. J. Milburn, Quantum Optics (SpringerVerlag, Berlin, 1994).

[10] B. J. Oliver and C. R. Stroud, Jr., Phys. Lett. A 135, 407 (1989); S. M. Tan, M. J. Holland, and D. F. Walls, Opt. Commun. 77, 285 (1990); S. M. Tan, D. F. Walls, and M. J. Collett,
Phys. Rev. Lett. 66, 252 (1991); L. Hardy, ibid. 73, 2279 (1994).

[11] Y. S. Kim and M. E. Noz, Phase Space Picture of Quantum Mechanics (World Scientific, Singapore, 1991).

[12] J. G. Rarity, Ann. (N.Y.) Acad. Sci. 755, 624 (1995); P. Hariharan, N. Brown, and B. C. Sanders, J. Mod. Opt. , 40, 113 (1993); P. Hariharan, I. Fujima, N. Brown, and B. C. Sanders, ibid. 40, 1477 (1993); P. Hariharan, N. Brown, and B. C. Sanders, ibid. 40, 1573 (1993); P. Hariharan, I. Fujima, N. Brown, and B. C. Sanders, ibid. 42, 565 (1995).

[13] J. G. Walker and E. Jakeman, Opt. Acta 32, 1303 (1985); J. H. Jefferson and E. Jakeman, ibid. 33, 557 (1986); P. R. Tapster, J. G. Rarity, and P. C. M. Owens, Phys. Rev. Lett. 73, 1923 (1994); P. D. Townsend, J. G. Rarity, and P. R. Tapster, Electron. Lett. 29, 1291 (1993); 29, 634 (1993); J. G. Rarity, J. Burnett, P. R. Tapster, and R. Paschotta, Europhys. Lett. 22, 95 (1993); R. Y. Chiao, P. G. Kwiat, and A. M. Steinberg, Quantum Semiclass. Opt. 7, 259 (1995); P. G. Kwiat, P. H. Eberhard, A. M. Steinberg, and R. Y. Chiao, Phys. Rev. A 49, 3209 (1994). 